\title{
Classification of eye abnormality using statistical parameters in texture features of corneal arcus image
}

\begin{abstract}
The corneal arcus (CA), is the white-gray sediments, exist within the iris-limbus like a circle ring, caused by the occurrence of lipid disorder, in the bloodstream. This sign shows, the indication to diseases such as the coronary heart disease, diabetes, and hypertension. This paper demonstrates the classification of the CA as an indicator of hyperlipidemia. The experiment, uses two sets of sample data, consisting of the normal and abnormal eyes (i.e., CA), for classifies each group. The step for this classification, begin with the normalization of the eye images (as part of pre-processing), to achieve the region of interest (ROI). The next process is to extract the image texture using the grey level co-occurrence matrix (GLCM) technique, and calculate the extraction of the image texture using the statistical method. These features, then will be fed into the classifier, as the input for several processes, namely as the data training, data testing and validation data. In these experiments, we have obtained the excellent result using the proposed framework. This proves that, by using a Bayesian regularization (BR) classifier, the results of this classification given by the sensitivity (94\%), specificity (100\%), and accuracy (97.78\%). Applications/Improvements: Based on the results obtained, the proposed system is successfully to classify the images with the CA signs. This show that, this proposed method can be applied to identify the presence of the hypercholesterolemia in a noninvasive test, to classify and detect the image of the CA.
\end{abstract}

Keyword: Accuracy; Classifier; Corneal Arcus; Hyperlipidemia; Sensitivity; Specificity 